\title{
Synthesis of Palladium Nanoparticles by Reaction of Filamentous Cyanobacterial Biomass with a Palladium(II)- Chloride Complex
}

\author{
Maggy F. Lengke*, Michael E. Fleet, and Gordon Southam
}

Department of Earth Sciences, University of Western Ontario, London, Ontario, N6A 5B7, Canada

Corresponding Author. *E-mail: maggylengke@yahoo.com or maggylengke@comcast.net. Present address: Geomega Inc., 2995 Baseline Road, Suite 202, Boulder, Colorado 80303, USA

\begin{tabular}{|c|c|c|c|c|c|c|c|c|c|c|}
\hline Complex & Oxidation State & $\begin{array}{c}\text { Binding Energy } \\
\mathrm{Pd} 3 d 5 / 2 \\
(\mathrm{eV})\end{array}$ & Starting reagent & Reducing agent & $\begin{array}{c}\text { Oxidizing } \\
\text { agent }\end{array}$ & $\begin{array}{c}\text { Stabilizing } \\
\text { agent }\end{array}$ & Catalyst & Substrate & Size & Reference \\
\hline$\overline{\mathrm{Pd}}$ & 0 & 335.7 & & & & & & & & 39 \\
\hline $\mathrm{PdCl}_{2}$ & 2 & 337.9 & & & & & & & & 39 \\
\hline Mixture $P d$ and $P d$ & 0 and 2 & 337.9 & & & & & & & & 39 \\
\hline $\mathrm{PdBr}_{2}$ & 2 & 337.3 & & & & & & & & 39 \\
\hline $\mathrm{PdI}_{2}$ & 2 & 336.6 & & & & & & & & 39 \\
\hline $\operatorname{Pd}(\mathrm{CN})_{2}$ & 2 & 339.6 & & & & & & & & 39 \\
\hline $\mathrm{K}_{2} \mathrm{PdCl}_{4}$ & 2 & 338.4 & & & & & & & & 39 \\
\hline $\mathrm{K}_{2} \mathrm{PdBr}_{4}$ & 2 & 337.5 & & & & & & & & 39 \\
\hline $\mathrm{K}_{2} \mathrm{Pd}\left(\mathrm{NO}_{2}\right)_{4}$ & 2 & 339.2 & & & & & & & & 39 \\
\hline $\mathrm{K}_{2} \mathrm{Pd}(\mathrm{CN})_{4}$ & 2 & 339.2 & & & & & & & & 39 \\
\hline $\mathrm{Pd}\left(\mathrm{P}\left(\mathrm{C}_{6} \mathrm{H}_{5}\right)_{3}\right)_{2} \mathrm{Cl}_{2}$ & 2 & 337.9 & & & & & & & & 39 \\
\hline $\mathrm{Pd}\left(\mathrm{P}\left(\mathrm{C}_{6} \mathrm{H}_{5}\right)_{3}\right)_{2} \mathrm{Br}_{2}$ & 2 & 338 & & & & & & & & 39 \\
\hline $\mathrm{Pd}\left(\mathrm{P}\left(\mathrm{C}_{6} \mathrm{H}_{5}\right)_{3}\right)_{2} \mathrm{I}_{2}$ & 2 & 337.7 & & & & & & & & 39 \\
\hline $\operatorname{Pd}\left(\mathrm{P}\left(\mathrm{C}_{6} \mathrm{H}_{5}\right)_{3}\right)_{2}(\mathrm{CN})$ & 2 & 338.4 & & & & & & & & 39 \\
\hline $\mathrm{Pd}\left(\mathrm{NH}_{3}\right)_{4}{ }^{2+}$ & 2 & 337.6 & $\mathrm{Pd}\left(\mathrm{NH}_{3}\right)_{4}{ }^{2+}$ & & & & & Na-Y zeolite & & 40 \\
\hline Pd(II) & 2 & 339.2 & $\mathrm{Pd}\left(\mathrm{NH}_{3}\right)_{4}{ }^{2+}$ & oxygen gas & & & $500^{\circ} \mathrm{C}$ & $\mathrm{Na}-\mathrm{Y}$ zeolite & & 40 \\
\hline $\operatorname{Pd}(\mathrm{I})$ & 2 & 337.1 & $\mathrm{Pd}\left(\mathrm{NH}_{3}\right)_{4}{ }^{2+}$ & oxygen/hydrogen gas & & & $25 / 500^{\circ} \mathrm{C}$ & Na-Y zeolite & & 40 \\
\hline $\mathrm{Pd}$ & 0 & 336.4 & $\mathrm{Pd}\left(\mathrm{NH}_{3}\right)_{4}{ }^{2+}$ & hydrogen gas & & & $350^{\circ} \mathrm{C}$ & Na-Y zeolite & & 40 \\
\hline Pd & 0 & 335.2 & $\mathrm{Pd}\left(\mathrm{NH}_{3}\right)_{4}{ }^{2+}$ & hydrogen gas & & & $350^{\circ} \mathrm{C}$ & Na-Y zeolite & $2 \mathrm{~nm}$ & 40 \\
\hline $\mathrm{PdCl}_{2}$ & 2 & 338.2 & $\mathrm{PdCl}_{2}$ & & & & $100^{\circ} \mathrm{C}$ & $\mathrm{SiO}_{2}$ & & 41 \\
\hline $\mathrm{PdCl}_{2}$ & 2 & 337.4 & $\mathrm{PdCl}_{2}$ & & & & $100^{\circ} \mathrm{C}$ & $\mathrm{TiO}_{2}$ & & 41 \\
\hline $\mathrm{PdCl}_{2}$ & 2 & 338.5 & $\mathrm{PdCl}_{2}$ & & & & $100^{\circ} \mathrm{C}$ & $\mathrm{Al}_{2} \mathrm{O}_{3}$ & & 41 \\
\hline Pd & 0 & 335.5 & & & & & $100^{\circ} \mathrm{C}$ & & & 41 \\
\hline $\mathrm{Pd}$ & 0 & 335.6 & $\mathrm{PdCl}_{2}$ & hydrogen gas & & & $200^{\circ} \mathrm{C}$ & $\mathrm{SiO}_{2}$ & & 41 \\
\hline $\mathrm{Pd}$ & 2 & 335.6 & $\mathrm{PdCl}_{2}$ & hydrogen gas & & & $200^{\circ} \mathrm{C}$ & $\mathrm{TiO}_{2}$ & & 41 \\
\hline Pd & 2 & 335.6 & $\mathrm{PdCl}_{2}$ & hydrogen gas & & & $200^{\circ} \mathrm{C}$ & $\mathrm{Al}_{2} \mathrm{O}_{3}$ & & 41 \\
\hline Pd & 0 & 335.5 & & & & & $200^{\circ} \mathrm{C}$ & & & 41 \\
\hline $\mathrm{Pd}$ & 0 & 335.6 & $\mathrm{Pd}\left(\mathrm{NH}_{3}\right)_{4} \mathrm{Cl}_{2}$ & hydrogen gas & & & $300^{\circ} \mathrm{C}$ & $\mathrm{Al}_{2} \mathrm{O}_{3}$ & & 41 \\
\hline Pd & 0 & 335.7 & $\mathrm{Pd}\left(\mathrm{NO}_{3}\right)_{2}$ & hydrogen gas & & & $300^{\circ} \mathrm{C}$ & $\mathrm{Al}_{2} \mathrm{O}_{3}$ & & 41 \\
\hline $\mathrm{Pd}$ & 0 & 336.3 & $\mathrm{PdCl}_{2}$ & hydrogen gas & & & $300^{\circ} \mathrm{C}$ & $\mathrm{Al}_{2} \mathrm{O}_{3}$ & & 41 \\
\hline Pd & 0 & 335.6 & $\mathrm{Pd}\left(\mathrm{NH}_{3}\right)_{4} \mathrm{Cl}_{2}$ & hydrogen gas & & & $300^{\circ} \mathrm{C}$ & $\mathrm{SiO}_{2}$ & & 41 \\
\hline $\mathrm{Pd}$ & 0 & 335.6 & $\operatorname{Pd}\left(\mathrm{NO}_{3}\right)_{2}$ & hydrogen gas & & & $300^{\circ} \mathrm{C}$ & $\mathrm{SiO}_{2}$ & & 41 \\
\hline $\mathrm{Pd}$ & 0 & 335.9 & $\mathrm{PdCl}_{2}$ & hydrogen gas & & & $300^{\circ} \mathrm{C}$ & $\mathrm{SiO}_{2}$ & & 41 \\
\hline
\end{tabular}

Table 1. Binding energy of $\mathrm{Pd} 3 d_{5 / 2}$ XPS lines for $\mathrm{Pd}(0)$ and $\mathrm{Pd}(\mathrm{II})$ species. 
Table 1. Continued.

\begin{tabular}{|c|c|c|c|c|c|c|c|c|c|c|}
\hline Complex & Oxidation State & $\begin{array}{c}\text { Binding Energy } \\
\operatorname{Pd} 3 d 5 / 2 \\
(\mathrm{eV})\end{array}$ & Starting reagent & Reducing agent & $\begin{array}{c}\text { Oxidizing } \\
\text { agent }\end{array}$ & $\begin{array}{c}\text { Stabilizing } \\
\text { agent }\end{array}$ & Catalyst & Substrate & Size & Reference \\
\hline$\overline{\mathrm{Pd}\left(\mathrm{NO}_{3}\right)_{2}}$ & 2 & 337.1 & $\mathrm{Pd}\left(\mathrm{NO}_{3}\right)_{2}$ & & & & & $\mathrm{ZnO}$ & & 42 \\
\hline $\mathrm{PdCl}_{4}{ }^{2-}$ & 2 & 337.9 & $\mathrm{H}_{2} \mathrm{PdCl}_{4}$ & & & & & & & 43 \\
\hline $\mathrm{PdCl}_{2}\left(\mathrm{H}_{2} \mathrm{O}\right)_{2}$ & 2 & 337 & $\mathrm{H}_{2} \mathrm{PdCl}_{4}$ & & & & & & & 43 \\
\hline $\mathrm{Pd}\left(\mathrm{H}_{2} \mathrm{O}\right)_{4}{ }^{2+}$ & 2 & 335.9 & $\mathrm{H}_{2} \mathrm{PdCl}_{4}$ & & & & & & & 43 \\
\hline $\mathrm{Pd}$ & 0 & $335.1-335.4$ & $\mathrm{H}_{2} \mathrm{PdCl}_{4}$ & & & & & $\mathrm{SiO}_{2}$ & & 43 \\
\hline $\mathrm{H}_{2} \mathrm{PdCl}_{4}$ & 2 & 336.9 & $\mathrm{H}_{2} \mathrm{PdCl}_{4}$ & & & & & $\mathrm{La}_{2} \mathrm{O}_{3}$ & & 43 \\
\hline $\mathrm{H}_{2} \mathrm{PdCl}_{4}$ & 2 & 336.2 & $\mathrm{H}_{2} \mathrm{PdCl}_{4}$ & air & & & & $\mathrm{La}_{2} \mathrm{O}_{3}$ & & 43 \\
\hline $\mathrm{Pd}$ & 0 & 334.9 & $\mathrm{H}_{2} \mathrm{PdCl}_{4}$ & air & & & & $\mathrm{La}_{2} \mathrm{O}_{3}$ & & 43 \\
\hline $\mathrm{Pd}$ & 0 & 334.8 & $\mathrm{H}_{2} \mathrm{PdCl}_{4}$ & hydrogen gas & & & $300^{\circ} \mathrm{C}$ & $\mathrm{La}_{2} \mathrm{O}_{3}$ & & 43 \\
\hline $\mathrm{Pd}$ & 0 & 334.8 & $\mathrm{H}_{2} \mathrm{PdCl}_{4}$ & hydrogen gas & & & $400^{\circ} \mathrm{C}$ & $\mathrm{La}_{2} \mathrm{O}_{3}$ & & 43 \\
\hline $\mathrm{Pd}(\mathrm{II})$ & 2 & 336.8 & $\mathrm{Pd}\left(\mathrm{NH}_{3}\right)_{4} \mathrm{Cl}_{2}$ & & & & $25^{\circ} \mathrm{C}$ & Na-X zeolite & & 44 \\
\hline $\mathrm{Pd}\left(\mathrm{NH}_{3}\right)_{4} \mathrm{Cl}_{2}$ & 2 & 338.8 & $\mathrm{Pd}\left(\mathrm{NH}_{3}\right)_{4} \mathrm{Cl}_{2}$ & & & & $25^{\circ} \mathrm{C}$ & Na-X zeolite & & 44 \\
\hline $\mathrm{Pd}(\mathrm{II})$ & 2 & 337.4 & $\mathrm{Pd}\left(\mathrm{NO}_{3}\right)_{2}$ & & & & $25^{\circ} \mathrm{C}$ & $\mathrm{Na}-\mathrm{X}$ zeolite & & 44 \\
\hline $\mathrm{Pd}$ & 0 & $334.79(335.1)$ & & & & & & & & $45-46$ \\
\hline $\mathrm{PdO}$ & 2 & 336.3 & & & & & & & & $45-46$ \\
\hline $\mathrm{PdO}_{2}$ & 2 & 337.9 & & & & & & & & $45-46$ \\
\hline $\mathrm{PdI}_{2}$ & 2 & 337.1 & & & & & & & & 45 \\
\hline $\mathrm{PdBr}_{2}$ & 2 & 337.1 & & & & & & & & 45 \\
\hline $\mathrm{PdCl}_{2}$ & 2 & 337.8 & & & & & & & & 45 \\
\hline $\mathrm{K}_{2} \mathrm{Pd}\left(\mathrm{NO}_{2}\right)_{4}$ & 2 & 338.8 & & & & & & & & 45 \\
\hline $\mathrm{K}_{2} \mathrm{PdBr}_{4}$ & 2 & 337.7 & & & & & & & & 45 \\
\hline $\mathrm{K}_{2} \mathrm{PdCl}_{4}$ & 2 & 337.9 & & & & & & & & 45 \\
\hline $\mathrm{Pd}\left(\mathrm{NH}_{3}\right)_{4} \mathrm{Cl}_{2}$ & 2 & 338.4 & & & & & & & & 45 \\
\hline $\mathrm{Pd}(\mathrm{OAc})_{2}$ & 2 & 338.6 & & & & & & & & $45-46$ \\
\hline $\mathrm{Pd}$ & 0 & $335.7-335.9$ & $\mathrm{Pd}(\mathrm{hfac})_{2}$ & & & & & $\mathrm{Cu}$ & & 47 \\
\hline $\operatorname{Pd}(h f a c)_{2}$ & 2 & 339.1 & & & & & & & & 47 \\
\hline $\mathrm{Pd}$ & 0 & 335.7 & $\operatorname{Pd}\left(\eta_{3}-\mathrm{C}_{3} \mathrm{H}_{5}\right)(\mathrm{hfa})$ & helium $/ 1 \%$ hydrogen gas & & & $45-60^{\circ} \mathrm{C}$ & glass & $300-1000 \mathrm{nr}$ & 48 \\
\hline $\mathrm{Pd}$ & 0 & 335.7 & $\mathrm{Pd}\left(\eta_{3}-\mathrm{C}_{3} \mathrm{H}_{5}\right)(\mathrm{Cp})$ & helium $/ 1 \%$ hydrogen gas & & & $30-60^{\circ} \mathrm{C}$ & glass & $300-1000 \mathrm{nr}$ & 48 \\
\hline $\mathrm{Pd}$ & 0 & 336 & $\mathrm{C}_{51} \mathrm{H}_{42} \mathrm{O}_{3} \mathrm{Pd}_{2}$ & mesitylene & & & & & & 49 \\
\hline $\mathrm{C}_{51} \mathrm{H}_{42} \mathrm{O}_{3} \mathrm{Pd}_{2}$ & 0 & 335.2 & & & & & & & & 49 \\
\hline Pd foil & 0 & 334.8 & & & & & & & & 50 \\
\hline Pd film & 0 & 334.8 & & & & & & $\mathrm{SiO}_{2}$ & $500 \mathrm{~nm}$ & 50 \\
\hline Pd film & 0 & 334.8 & & & & & & $\mathrm{SiO}_{2}$ & $10 \mathrm{~nm}$ & 50 \\
\hline Pd dots & 0 & 335.8 & & & & & & $\mathrm{SiO}_{2}$ & $7 \mathrm{~nm}$ & 50 \\
\hline Pd pits & 0 & 335.9 & & & & & & $\mathrm{SiO}_{2}$ & $20 \mathrm{~nm}$ & 50 \\
\hline Pd dots & 0 & 335.9 & & & & & & $\mathrm{SiO}_{2}$ & $20 \mathrm{~nm}$ & 50 \\
\hline Pd foil & 0 & 334.8 & & argon/hydrogen gas & oxygen gas & & $400^{\circ} \mathrm{C}$ & & & 50 \\
\hline Pd film & 0 & 334.8 & & argon/hydrogen gas & oxygen gas & & $400^{\circ} \mathrm{C}$ & $\mathrm{SiO}_{2}$ & $500 \mathrm{~nm}$ & 50 \\
\hline Pd film & 0 & 336 & & argon/hydrogen gas & oxygen gas & & $400^{\circ} \mathrm{C}$ & $\mathrm{SiO}_{2}$ & $10 \mathrm{~nm}$ & 50 \\
\hline Pd dots & 0 & 336 & & argon/hydrogen gas & oxygen gas & & $400^{\circ} \mathrm{C}$ & $\mathrm{SiO}_{2}$ & $7 \mathrm{~nm}$ & 50 \\
\hline Pd pits & 0 & 336 & & argon/hydrogen gas & oxygen gas & & $400^{\circ} \mathrm{C}$ & $\mathrm{SiO}_{2}$ & $20 \mathrm{~nm}$ & 50 \\
\hline Pd dots & 0 & 336 & & argon/hydrogen gas & oxygen gas & & $400^{\circ} \mathrm{C}$ & $\mathrm{SiO}_{2}$ & $20 \mathrm{~nm}$ & 50 \\
\hline Pd foil & 0 & 336.6 & & argon/hydrogen gas & oxygen gas & & $600^{\circ} \mathrm{C}$ & & & 50 \\
\hline Pd film & 0 & 336.5 & & argon/hydrogen gas & oxygen gas & & $600^{\circ} \mathrm{C}$ & $\mathrm{SiO}_{2}$ & $500 \mathrm{~nm}$ & 50 \\
\hline Pd film & 0 & 337.9 & & argon/hydrogen gas & oxygen gas & & $600^{\circ} \mathrm{C}$ & $\mathrm{SiO}_{2}$ & $10 \mathrm{~nm}$ & 50 \\
\hline Pd dots & 0 & 337.9 & & argon/hydrogen gas & oxygen gas & & $600^{\circ} \mathrm{C}$ & $\mathrm{SiO}_{2}$ & $7 \mathrm{~nm}$ & 50 \\
\hline Pd pits & 0 & 337.9 & & argon/hydrogen gas & oxygen gas & & $600^{\circ} \mathrm{C}$ & $\mathrm{SiO}_{2}$ & $20 \mathrm{~nm}$ & 50 \\
\hline Pd dots & 0 & 337.9 & & argon/hydrogen gas & oxygen gas & & $600^{\circ} \mathrm{C}$ & $\mathrm{SiO}_{2}$ & $20 \mathrm{~nm}$ & 50 \\
\hline $\mathrm{PdO}_{2}$ & 2 & $337.6-337.8$ & $\mathrm{PdCl}_{2}$ & & & & $\mathrm{CeO}_{2}$ & $\mathrm{Al}_{2} \mathrm{O}_{3}$ & & 51 \\
\hline $\mathrm{Pd}$ & 0 & 335.4 & $\mathrm{PdCl}_{2}$ & & & & $\mathrm{CeO}_{2}$ & $\mathrm{Al}_{2} \mathrm{O}_{3}$ & & 51 \\
\hline $\mathrm{PdO}$ & 2 & 336.9 & $\mathrm{PdCl}_{2}$ & & & & & $\mathrm{Al}_{2} \mathrm{O}_{3}$ & & 51 \\
\hline $\mathrm{Pd}$ & 0 & 335.2 & $\mathrm{PdCl}_{2}$ & & & & & $\mathrm{Al}_{2} \mathrm{O}_{3}$ & & 51 \\
\hline $\mathrm{Pd}$ & 0 & 335.9 & $\mathrm{H}_{2} \mathrm{PdCl}_{2}$ & & & & $20^{\circ} \mathrm{C}$ & AISI 316 stainless steel & $140 \mathrm{~nm}$ & 52 \\
\hline $\mathrm{PdO}$ & 2 & 336.5 & $\mathrm{Pd}\left(\mathrm{CH}_{3}-\mathrm{COO}\right)_{2}$ & $\mathrm{NH}_{3}$ & & SBA-15 & & & $1.1-3.0 \mathrm{~nm}$ & 53 \\
\hline $\operatorname{Pd}\left(\eta^{5}-\mathrm{Cp}\right)\left(\eta^{3}\right.$-allyl & 2 & 337.7 & & & & & & $\operatorname{Pd}(111)$ & & 54 \\
\hline Pd & 0 & 335.6 & $\mathrm{H}_{2} \mathrm{PdCl}_{2}$ & $\mathrm{NH}_{2} \mathrm{NH}_{2}$ & & poly(vinylpyridine) & & & $2-4 \mu \mathrm{m}$ & 55 \\
\hline $\mathrm{Pd}$ & 0 & 335.1 & $\mathrm{PdCl}_{2}$ & cyanobacteria & & & $60-100^{\circ} \mathrm{C}$ & & $\leq 30 \mathrm{~nm}$ & This study \\
\hline $\mathrm{PdCl}_{2}$ & 2 & 338 & $\mathrm{PdCl}_{2}$ & cyanobacteria & & & $60-100^{\circ} \mathrm{C}$ & & & This study \\
\hline Pd-organic & & 336 & $\mathrm{PdCl}_{2}$ & cyanobacteria & & & $25^{\circ} \mathrm{C}$ & & $\leq 20 \mathrm{~nm}$ & This study \\
\hline $\mathrm{PdH}_{0.32}$ and $\mathrm{Pd}$ & & 335.8 & $\mathrm{PdCl}_{2}$ & & & & $100^{\circ} \mathrm{C}$ & & $<1 \mu \mathrm{m}$ & This study \\
\hline
\end{tabular}

